\title{
Maternal immunization with ovalbumin or Dermatophagoides pteronyssinus has opposing effects on FcyRllb expression on offspring B cells
}

Aline Aparecida de Lima Lira, Marília Garcia de Oliveira, Luana Mendonça de Oliveira, Alberto José da Silva Duarte, Maria Notomi Sato and Jefferson Russo Victor ${ }^{*}$

\begin{abstract}
Background: Over the last decade, our group has demonstrated that murine preconception immunization with allergens has a protective effect on allergy development in offspring. The murine model used in the present study allowed us to compare allergy induction by ovalbumin (OVA) and dust mite extract from Dermatophagoides pteronyssinus (Dp).

Findings: Female mice were immunized with OVA or Dp. Pups from immunized and non-immune mothers were immunized at 3 days old (do) with the same antigen used for the maternal immunization. The offspring were analyzed at 20 do. Preconceptional immunization with OVA or Dp did not increase maternal IgE serum levels, although the immunizations induced an increase in allergen-specific lgG1 Ab levels. Offspring serum analyses revealed that maternal immunization with OVA suppressed IgE production only in offspring immunized with OVA. Both preconception immunization protocols inhibited cellular influx into the airways of immunized offspring compared with controls. Similar frequencies of offspring IgM + B cells were found in the OVA- and Dp-immunized groups compared with their respective control groups. Moreover, preconception immunization with OVA enhanced FcyRllb expression on OVA-immunized offspring B cells. In contrast, decreased FcyRllb expression was detected on Dp-immunized offspring B cells compared with cells from the offspring of non-immune mothers.
\end{abstract}

Conclusions: Together, these results show that preconception OVA immunization and Dp immunization can inhibit allergy development but have opposite effects on FcyRllb expression on offspring B cells.

Keywords: Allergy, Maternal immunization, Dermatophagoides pteronyssinus, FcyRllb

\section{Introduction}

In an earlier study, we showed that preconception immunization with the mite Dermatophagoides pteronyssinus $(\mathrm{Dp})$ could suppress anaphylactic IgE production and regulate Th2 cytokine exacerbation in offspring [1]. Subsequently, we observed that preconception immunization with ovalbumin (OVA) could induce the passive transference of maternal anti-OVA IgG1 antibodies (Abs) at high levels [2], which could be detected in the sera of offspring concomitant with the increased expression of inhibitory FcyRIIb receptors on B cells [3].

\footnotetext{
* Correspondence: victorjr@usp.br

Laboratory of Dermatology and Immunodeficiencies, LIM-56, University of São Paulo, Medical School, Av. Dr. Enéas de Carvalho Aguiar, 500, 3rd floor, 05403-000 São Paulo, Brazil
}

Co-localizing with B cell receptors (BCRs), FcyRIIb receptors can interact with IgG/antigen immune complexes and phosphorylate the inhibitory phosphatase SHIP, leading to the inhibition of B cell activation [4]. This process hinders formation of the immunological synapse between $B$ cells and $\mathrm{CD} 4+\mathrm{T}$ cells, which is necessary for isotype switching and IgE production [5].

Our hypothesis was that maternal antibodies (MatAbs) transferred during pregnancy and breastfeeding can form immune complexes with allergens from offspring and inhibit the activation of offspring B cells. In humans, the increased passive transfer of anti-Dp MatAbs does not have a protective effect on offspring [6]. Although OVA is more widely used in murine models of type I hypersensitivity, Dp is an important commonly inhaled allergen that 
causes bronchial asthma and allergic rhinitis in humans [7]. To date, the effect of maternal immunization with $\mathrm{Dp}$ on the expression of inhibitory receptors in offspring $B$ cells has not been evaluated. In this study, we use a murine model to compare the effects of OVA and Dp immunization to better understand neonatal allergy regulation and to guide future studies of allergy regulation in humans.

\section{Methods}

Mice

Male and female C57BL/6 inbred mice were used at 8 to 10 weeks of age. Animals were purchased from the Central Animal Facility of the School of Medicine, University of Sao Paulo. Offspring were used during the neonatal period ( 3 days old (do)). All experiments described $\mathrm{n}$ this manuscript were approved by the University of Sao Paulo - School of Medicine - Animal Ethics Committee (CEP-FMUSP: 097/11 - Sao Paulo, SP, Brazil).

\section{Immunization protocols}

Female mice were immunized subcutaneously with 1500 $\mu \mathrm{g}$ OVA (Sigma, USA) or $10 \mu \mathrm{g}$ Dp (Indoor Biotechnologies, USA) in $6 \mathrm{mg}$ Alum (FURP, Sao Paulo) and boosted after 10 and 20 days with $1000 \mu \mathrm{g}$ OVA or $10 \mu \mathrm{g}$ Dp in saline intraperitoneally (i.p.). Females were mated at 21 days post immunization. The pups of immunized and non-immune mothers were immunized with the same antigen used for maternal immunization. Offspring at 3 do were immunized (i.p.) with $100 \mu \mathrm{g}$ OVA or $10 \mu \mathrm{g}$ Dp in 0.6 mg Alum and boosted after 10 days with the same antigen/ dose in saline. Sera from the mothers were obtained at term. Experimental analyses of the offspring were performed at 20 do. As a control group, non-immunized offspring from non-immune mothers were bled at $20 \mathrm{do}$, and the sera were analyzed for total IgE production.

\section{Determination of total IgE and anti-OVA/Dp lgG1 Ab levels}

OVA- and Dp-specific IgG1 and total IgE antibodies were measured by ELISA, as previously described [2]. To measure total IgE, a standard curve was used (Pharmingen, USA). The anti-OVA and anti-Dp Ab levels are expressed as optical densities.

\section{Lung inflammation}

Offspring from either immune or non-immune mothers were immunized and subjected to nasal instillations with $100 \mu \mathrm{g}$ OVA or $10 \mu \mathrm{g}$ Dp at 43, 50, 57, 58 and 59 do. Bronchoalveolar fluid (BAL) was analyzed at 60 do following exsanguination of the abdominal aorta. The BAL was obtained by washing the lungs with three times with 1.5 mL PBS using a tracheal tube, which was then centrifuged at $800 \mathrm{rpm}$ for $10 \mathrm{~min}$. The cell pellet was
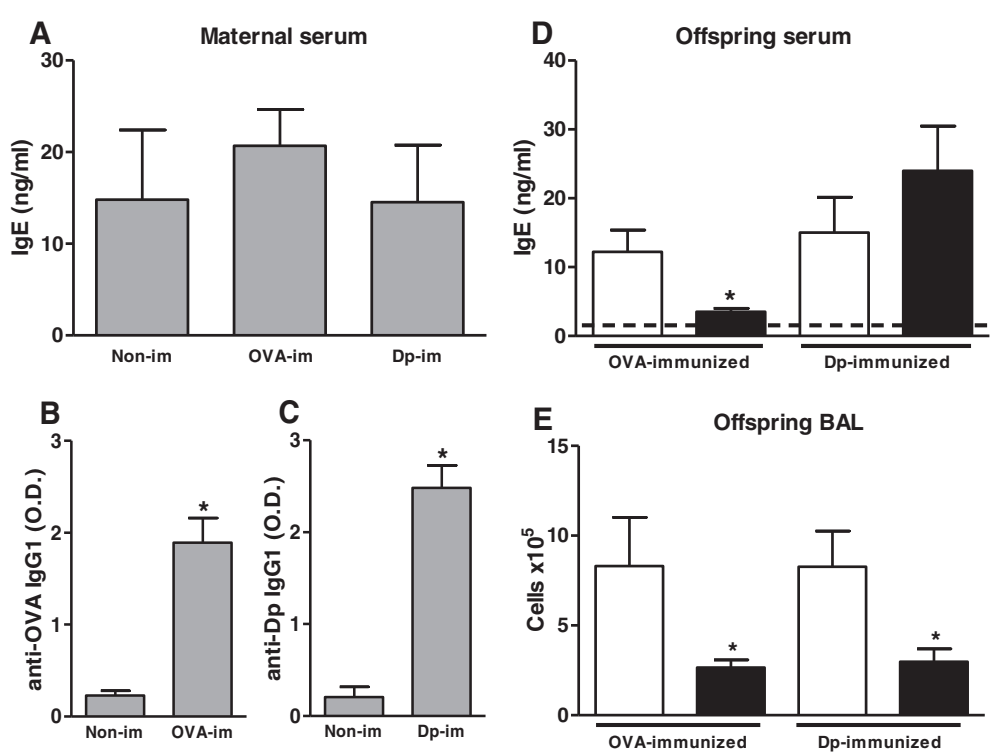

Figure 1 Decreased pulmonary inflammation in offspring in response to preconception OVA or Dp immunization. Female mice were immunized with OVA or Dp, boosted 10 and 20 days later, and mated on day 21. Sera from full-term pregnancies were evaluated for (A) total IgE, (B) anti-OVA IgG1 and (C) anti-Dp IgG1 Ab levels by ELISA. Sera from full-term pregnancies of non-immune females were assessed as controls. Offspring at 3 do were immunized with the same antigen used for the maternal immunizations, boosted at 13 do, and bled at 20 do. The offspring of control non-immune mothers were immunized with OVA or Dp as controls. Total IgE levels were determined by ELISA, and the dashed line represents IgE levels in control non-immunized 20 do offspring from nom-immune mothers (D). At 43 do, the offspring received five intranasal challenges with OVA or Dp. After 24 h, BAL was assessed, and total cell counts were evaluated (E). The data are presented as mean (8 to 10 mice per group) \pm SEM values. ${ }^{*} P \leq 0.05$ compared with the control group. 


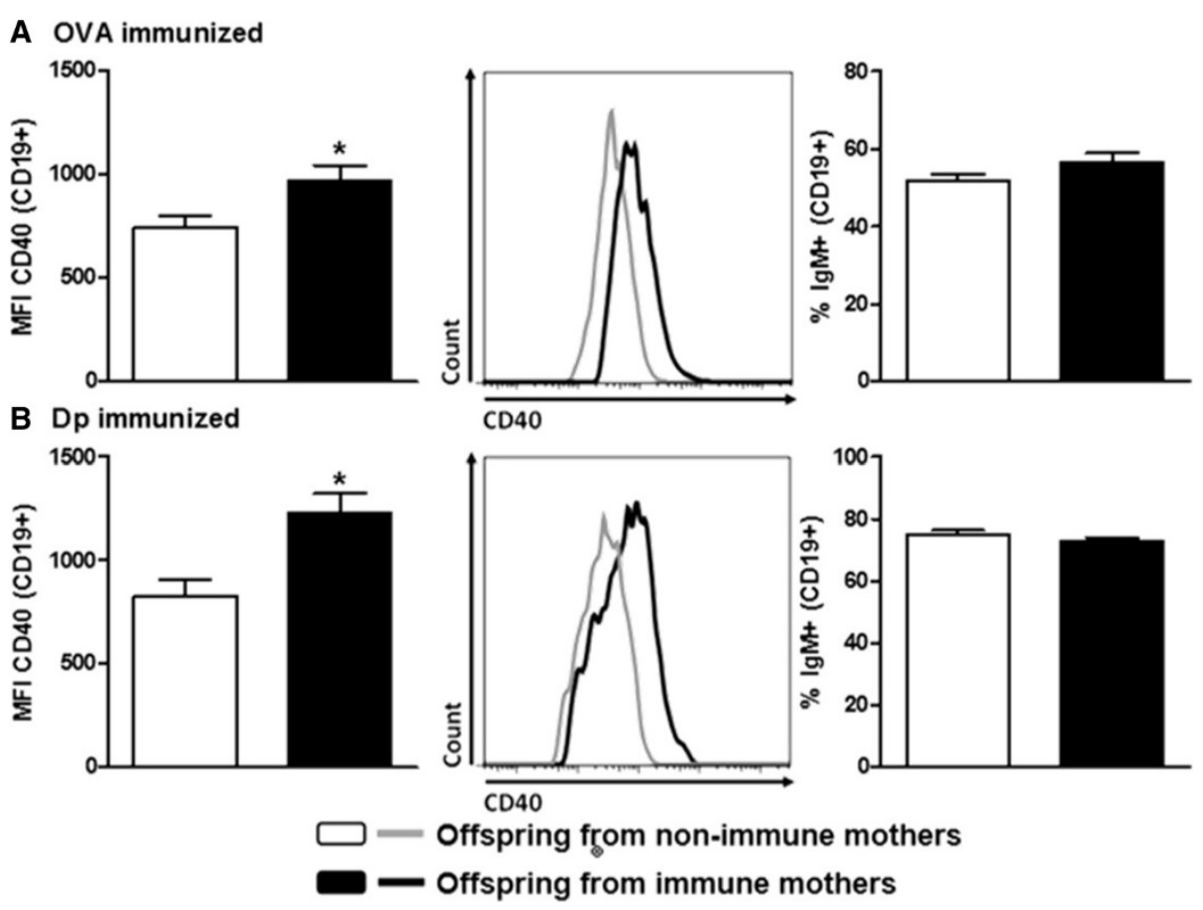

Figure 2 Increased CD40 expression on offspring B cells induced by maternal immunization. Offspring from (A) OVA- or (B) Dp-immunized mothers were immunized at 3 do with the same antigen used in the maternal immunization and boosted at 13 do. At 20 do, splenic CD19+ B cells were assessed for CD40 expression and the percentage of CD19+ $\operatorname{lgM}+$ cells. The control group was composed of offspring from non-immune mothers immunized with OVA or Dp. Representative histograms of CD40 expression for each group are shown (A-B, middle panels). The data are presented as mean (8 to 10 mice per group) \pm SEM values. ${ }^{*} P \leq 0.05$ compared with the control group.

diluted in $300 \mu \mathrm{L}$ PBS, and total leukocyte counts were performed using a Neubauer chamber.

\section{Spleen cell suspension}

Spleens were collected, and cells were harvested for flow cytometric analysis, as previously described [3].

\section{Flow cytometry}

For surface staining, a single-cell suspension in PBS + $1 \%$ BSA was incubated with the following fluorescentconjugated Abs (BD Biosciences, USA): anti-CD19, anti-CD40, anti-IgM or anti-CD16/32. Gating of the cells was based on specific isotype control values. Data were acquired using an LSRFortessa cytometer (BD Biosciences, USA) and analyzed with the Flowjo software program (Tree Star, USA).

\section{Statistical analysis}

Differences between groups were considered significant when $P$ values were $<0.05$ according to the MannWhitney test.

\section{Findings}

First, we assessed maternal serum Ab levels at full-term pregnancy after preconception immunization with OVA or Dp. Preconception immunization with OVA or Dp did not increase serum IgE levels (Figure 1A), although the immunizations did increase the levels of allergenspecific IgG1 Abs (Figures 1B-C). All immunized groups produced higher levels of total IgE compared with control non-immune offspring from non-immune mothers (Figure 1D). Analysis of the sera from the immunized offspring revealed that maternal immunization with OVA suppressed IgE production only in offspring immunized with OVA (Figure 1D) but not with Dp (Figure 1D).

Moreover, we evaluated the effect of maternal allergen immunization on pulmonary inflammation in offspring, and we verified that both preconception immunization protocols inhibited cellular influx into the airways of immunized offspring compared with offspring from nonimmune mothers (Figure 1E).

To verify the effect of maternal immunization on offspring B cells, we evaluated CD40 and Fc $\gamma$ RIIb expression on these cells. We observed that both maternal immunization protocols could upregulate CD40 expression on offspring $B$ cells compared with $B$ cells from the control offspring groups (Figure 2A). Similar frequencies of offspring IgM + B cells were found in the OVA- and Dp-immunized groups compared with their respective control groups (Figure 2B). Moreover, preconception immunization with OVA enhanced FcyRIIb expression on OVA-immunized offspring B cells compared with B 


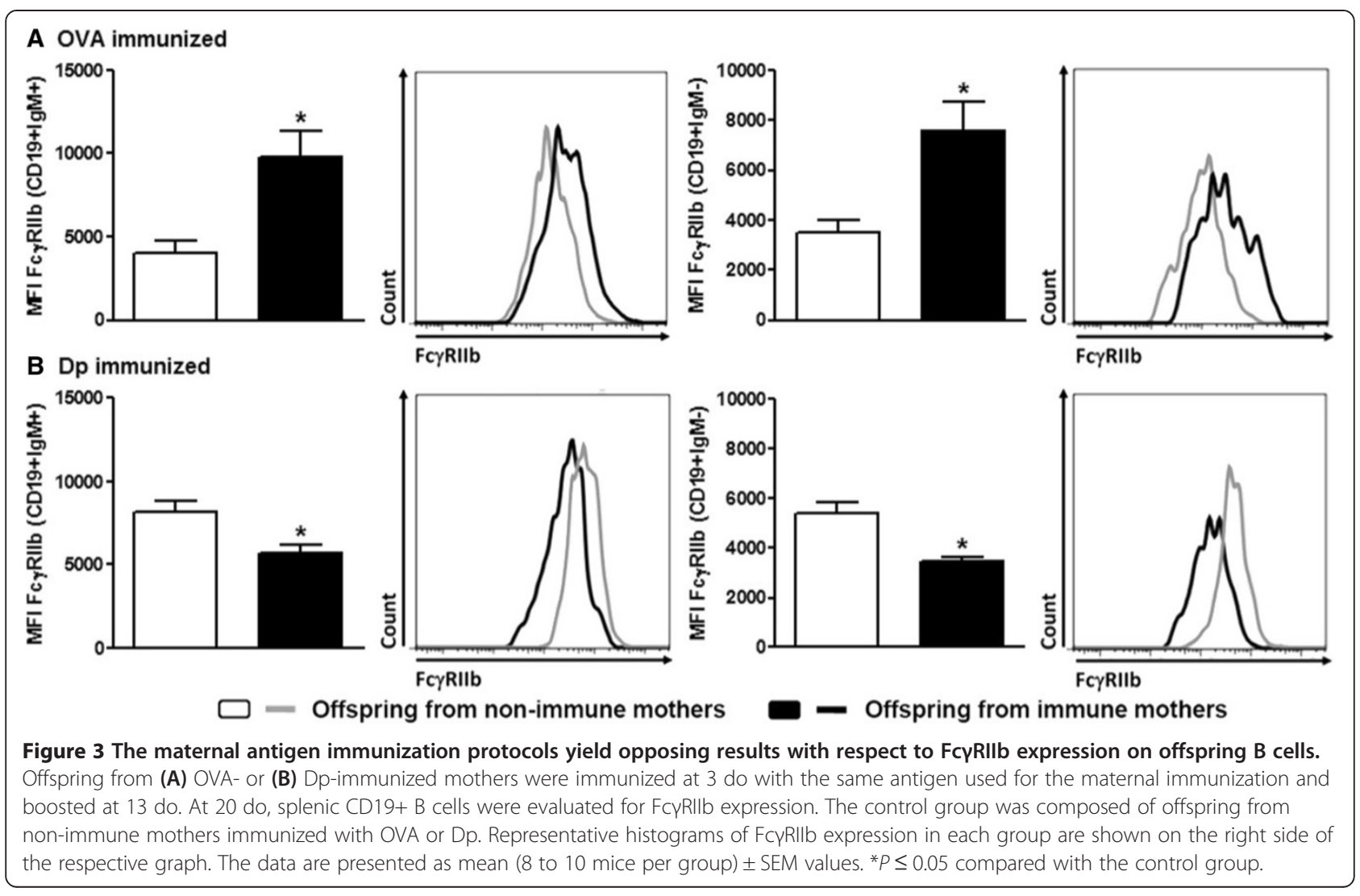

cells from OVA-immunized offspring from non-immune mothers (Figure 3A). In contrast, decreased FcyRIIb expression was observed on Dp-immunized offspring $B$ cells compared with B cells from the Dp-immunized offspring control group (Figure 3B).

\section{Discussion}

By varying the allergen dose in the maternal immunization protocol from 1 to $10 \mu \mathrm{g} \mathrm{Dp}$, we were able to detect an increase in the maternal $\mathrm{Ab}$ response and passive $\mathrm{Ab}$ transference in the highest titers $(10 \mu \mathrm{g})$ (data not shown). This dose could also inhibit the allergic response and lung inflammation in offspring and was therefore adopted as the standard dose.

Neither preconception immunization protocol induced higher total IgE levels, suggesting that the females did not reach allergic status and that both protocols could sensitize the maternal immune system. As we previously described, MatAbs are passively transferred to their offspring, concomitant with anaphylactic IgE suppression in offspring [1-3]. Preconception OVA immunization could reduce total IgE production in offspring, in contrast with maternal Dp immunization. However, both protocols could downregulate pulmonary inflammation in offspring, suggesting that allergy development in the offspring was inhibited by preconception OVA or Dp immunization.
Phenotypic analysis of offspring B cells revealed an equivalent increase in CD40 expression on offspring B cells from both types of immune mothers. However, this status could not have influenced isotype switching of the offspring $B$ cells in response to neonatal immunization, as the percentages of IgM $+B$ cells were similar between pups from OVA- or DP-immunized mothers compared with those from non-immune mothers.

Maternal OVA immunization can induce the upregulation of FcyRIIb receptors on offspring B cells at 20 do, as described previously by our group [3]. The inhibitory co-receptor Fc $\gamma$ RIIb can inhibit the in vitro activation of B cells $[4,8,9]$ and induce apoptosis in plasma cells [10]. Therefore, the upregulation of FcyRIIb levels on B cells in the presence of immune complexes may represent an effective way to downregulate immune activation. We detected the overexpression of FcyRIIb on offspring IgM + B cells, which consisted primarily of naïve B cells. This result is consistent with our hypothesis that passively transferred MatAbs can negatively regulate offspring $B$ cells by interacting with allergens in the offspring and inhibiting offspring $\mathrm{B}$ cell activation and isotype switching, thereby downregulating IgE production and allergy development. Our results demonstrate that this effect cannot be induced by maternal $\mathrm{Dp}$ immunization, for which an opposite effect was observed, with offspring B cells 
showing downregulated FcyRIIb expression in response to maternal Dp immunization.

Our findings suggest that maternal Dp immunization does control offspring allergy development but without the upregulation of FcyRIIb on offspring B cells, as observed in the maternal OVA immunization protocol [3]. This phenomenon could be due to the fact that the Dp extract contains a variety of proteins with biological activity, in contrast with purified OVA. The main antigenic fraction of $\mathrm{Dp}$ (Der p 1) is a $25 \mathrm{kDa}$ cysteine protease. Recombinant Der p 1 and natural Der p 1 have similar biological effects on immune cells [11]. These effects include the optimization of antigen capture and processing by APCs [12], as well as the cleavage of CD23 on B cells $[13,14]$ and of CD25 on T cells [15]. This activity blocks the negative feedback mediated by IgE Abs that interact with $\mathrm{B}$ cells via $\mathrm{CD} 23$ and the induction of low IFN- $\gamma$ and high IL-4 production due to $\mathrm{CD} 25$ cleavage on $\mathrm{CD} 4+\mathrm{T}$ cells [16].

The regulation of FcyRIIb expression on B cells in response to CD23 activity has not been described, although it is possible that $\mathrm{Dp}$ antigenic activity may indirectly influence FcyRIIb expression. The fact that maternal Dp immunization can inhibit pulmonary inflammation in offspring, independent of FcyRIIb upregulation on offspring B cells, may be due to other mechanisms, such as allergen neutralization and idiotypic interactions [17] or the induction of offspring regulatory $\mathrm{T}$ cells [18]. In any case, the activity of these mechanisms appears to result in a similar mode of regulation in offspring until 20 do in mice, as we show in this report. Future studies on preconception Dp immunization should examine purified Dp proteins with similar structural and biological activities as the Dp extract.

\section{Competing interests}

The authors declare that they have no competing interest.

\section{Authors' contributions}

AALL carried out all experiments. MGO: cooperated with all experiments. LMO carried out flow cytometry acquisitions. AJSD participated in the design of the study. MNS participated in the design of the study and helped draft the manuscript. JRV conceived of the study, participated in its design and coordination, and helped draft the manuscript. All authors have read and approved the final manuscript.

\section{Acknowledgments}

The authors would like to thank the Fundação de Amparo à Pesquisa de São Paulo (FAPESP 2010/13262-5) and the LIM HC-FMUSP for their financial support.

Received: 2 April 2014 Accepted: 25 August 2014

Published: 2 September 2014

\section{References}

1. Victor JR Jr, Fusaro AE, Duarte AJ, Sato MN: Preconception maternal immunization to dust mite inhibits the type I hypersensitivity response of offspring. J Allergy Clin Immunol 2003, 111:269-277.

2. Fusaro AE, de Brito CA, Rigado PO, Goldoni AL, Duarte AJS, Sato MN: Maternal-fetal interaction: preconception immunization in mice prevents neonatal sensitization induced by allergen exposure during pregnancy and breastfeeding. Immunology 2007, 122:107-115.
3. Victor JR, Muniz BP, Fusaro AE, de Brito CA, Taniguchi EF, Duarte AJ, Sato $M N$ : Maternal immunization with ovalbumin prevents neonatal allergy development and up-regulates inhibitory receptor Fc gamma RIIB expression on B cells. BMC Immunol 2010, 11:11.

4. Muta T, Kurosaki T, Misulovin Z, Sanchez M, Nussenzweig MC, Ravetch JV: A 13-amino-acid motif in the cytoplasmic domain of Fc gamma RIIB modulates B-cell receptor signalling. Nature 1994, 369:340.

5. Sohn HW, Tolar P, Pierce SK: Membrane heterogeneities in the formation of $B$ cell receptor-Lyn kinase microclusters and the immune synapse. J Cell Biol 2008, 182:367-379.

6. Macchiaverni P, Arslanian C, Frazao JB, Palmeira P, Russo M, Verhasselt V, Condino-Neto A: Mother to child transfer of $\lg G$ and $\lg A$ antibodies against Dermatophagoides pteronyssinus. Scand J Immunol 2011, 74:619-627.

7. Voorhorst R, Spieksma-Boezeman MI, Spieksma FT: Is a Mite (Dermatophagoides Sp.) the Producer of the House-Dust Allergen? Allerg Asthma (Leipz) 1964, 10:329-334.

8. Amigorena S, Salamero J, Davoust J, Fridman WH, Bonnerot C: Tyrosine-containing motif that transduces cell activation signals also determines internalization and antigen presentation via type III receptors for IgG. Nature 1992, 358:337-341.

9. Brauweiler AM, Cambier JC: Fc gamma RIIB activation leads to inhibition of signalling by independently ligated receptors. Biochem Soc Trans 2003, 31:281-285.

10. Xiang Z, Cutler AJ, Brownlie RJ, Fairfax K, Lawlor KE, Severinson E, Walker EU, Manz RA, Tarlinton DM, Smith KG: FcgammaRllb controls bone marrow plasma cell persistence and apoptosis. Nat Immunol 2007, 8:419-429.

11. Herman J, Thelen N, Smargiasso N, Mailleux AC, Luxen A, Cloes M, De Pauw E, Chevigne A, Galleni M, Dumez ME: Der $p 1$ is the primary activator of Der $p$ 3, Der $p 6$ and Der $p 9$ the proteolytic allergens produced by the house dust mite Dermatophagoides pteronyssinus. Biochim Biophys Acta 1840, 2013:1117-1124.

12. Wan H, Winton HL, Soeller C, Tovey ER, Gruenert DC, Thompson PJ, Stewart GA, Taylor GW, Garrod DR, Cannell MB, Robinson C: Der $\mathrm{p} 1$ facilitates transepithelial allergen delivery by disruption of tight junctions. J Clin Invest 1999, 104:123-133.

13. Hewitt CR, Brown AP, Hart BJ, Pritchard DI: A major house dust mite allergen disrupts the immunoglobulin $E$ network by selectively cleaving CD23: innate protection by antiproteases. J Exp Med 1995, 182:1537-1544.

14. Schulz O, Laing P, Sewell HF, Shakib F: Der p I, a major allergen of the house dust mite, proteolytically cleaves the low-affinity receptor for human IgE (CD23). Eur J Immunol 1995, 25:3191-3194.

15. Schulz O, Sewell HF, Shakib F: Proteolytic cleavage of CD25, the alpha subunit of the human $T$ cell interleukin 2 receptor, by Der $p 1$, a major mite allergen with cysteine protease activity. J Exp Med 1998, 187:271-275.

16. Ghaemmaghami AM, Robins A, Gough L, Sewell HF, Shakib F: Human T cell subset commitment determined by the intrinsic property of antigen: the proteolytic activity of the major mite allergen Der $p 1$ conditions T cells to produce more IL-4 and less IFN-gamma. Eur J Immunol 2001, 31:1211-1216.

17. Rigato PO, Fusaro AE, Victor JR, Sato MN: Maternal immunization to modulate the development of allergic response and pathogen infections. Immunotherapy 2009, 1:141-156

18. Mosconi E, Rekima A, Seitz-Polski B, Kanda A, Fleury S, Tissandie E, Monteiro R, Dombrowicz DD, Julia V, Glaichenhaus N, Verhasselt V: Breast milk immune complexes are potent inducers of oral tolerance in neonates and prevent asthma development. Mucosal Immunol 2010, 3:461-474.

doi:10.1186/1710-1492-10-47

Cite this article as: Lira et al:: Maternal immunization with ovalbumin or Dermatophagoides pteronyssinus has opposing effects on FcyRllb expression on offspring B cells. Allergy, Asthma \& Clinical Immunology 2014 10:47 